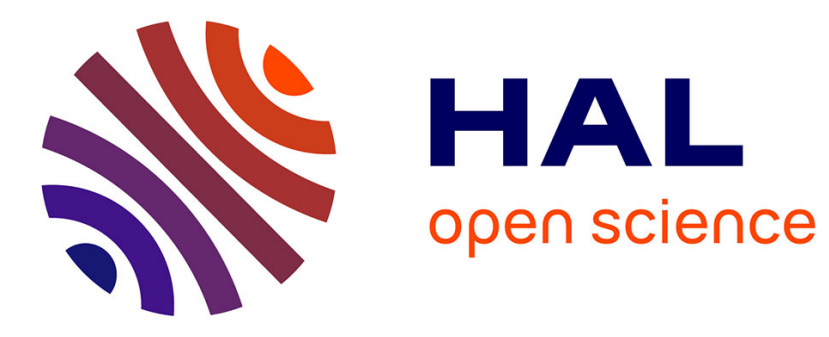

\title{
Energy-Aware Competitive Link Adaptation in Small-Cell Networks
}

Giacomo Bacci, Veronica Belmega, Panayotis Mertikopoulos, Luca Sanguinetti

\section{To cite this version:}

Giacomo Bacci, Veronica Belmega, Panayotis Mertikopoulos, Luca Sanguinetti. Energy-Aware Competitive Link Adaptation in Small-Cell Networks. 12th International Symposium on Modeling and Optimization in Mobile, Ad Hoc, and Wireless Networks (WiOpt), May 2014, Hammamet, Tunisia. 10.1109/wiopt.2014.6850272 . hal-01098846

\section{HAL Id: hal-01098846 https://hal.science/hal-01098846}

Submitted on 29 Dec 2014

HAL is a multi-disciplinary open access archive for the deposit and dissemination of scientific research documents, whether they are published or not. The documents may come from teaching and research institutions in France or abroad, or from public or private research centers.
L'archive ouverte pluridisciplinaire HAL, est destinée au dépôt et à la diffusion de documents scientifiques de niveau recherche, publiés ou non, émanant des établissements d'enseignement et de recherche français ou étrangers, des laboratoires publics ou privés. 


\title{
Energy-Aware Competitive Link Adaptation in Small-Cell Networks
}

\author{
(Invited Paper)
}

\author{
Giacomo Bacci*, E. Veronica Belmega ${ }^{\dagger}$, Panayotis Mertikopoulos ${ }^{\ddagger}$ and Luca Sanguinetti*§ \\ * Dipartimento di Ingegneria dell'Informazione, University of Pisa, Pisa, Italy, and CNIT, Parma, Italy \\ $\dagger$ ETIS / ENSEA - UCP - French National Center for Scientific Research (CNRS), Cergy-Pontoise, France \\ $\ddagger$ French National Center for Scientific Research (CNRS) and Laboratoire d’Informatique de Grenoble, France \\ $\S$ Alcatel-Lucent Chair, Ecole Supérieure d'Électricité (Supélec), Gif-sur-Yvette, France
}

\begin{abstract}
This work proposes a distributed power allocation scheme for maximizing the energy efficiency in the uplink of noncooperative small-cell networks based on orthogonal frequencydivision multiple-access technology. This is achieved by modeling users as rational agents that engage in a non-cooperative game in which every user selects the power loading so as to maximize his own utility (the user's throughput per Watt of transmit power) while satisfying minimum rate constraints. In this framework, we prove the existence of a Debreu equilibrium (also known as generalized Nash equilibrium) and we characterize the structure of the corresponding power allocation profile using techniques drawn from fractional programming. To attain this equilibrium in a distributed fashion, we also propose a method based on an iterative water-filling best response process. Numerical simulations are then used to assess the method's convergence and the performance of its end-state as a function of the system parameters.
\end{abstract}

\section{INTRODUCTION}

Small-cell networks are nowadays considered as one of the most promising solutions to address the seemingly contradictory future requirements of the Information and Communication Technology industry: more cellular network capacity and less energy consumption [1]. In a nutshell, the smallcell network concept amounts to a very dense deployment of operator-installed low-cost and low-power base stations equipped with advanced self-organization capabilities. This paradigm shift from carefully planned cellular networks to irregularly deployed self-optimizing base stations with different coverage makes the cellular architecture increasingly complex and heterogeneous, and poses many challenging issues to efficient network operation.

One of the key technical challenges in the deployment of small-cell networks is the involved network interference. A promising solution to this problem is commonly referred to as distributed cooperation [1], and aims at finding algorithmic solutions that approach the ideal cooperative gains while exploiting mostly local information and requiring limited interactions.

The research leading to these results has received funding from the People Programme (Marie Curie Actions) of the European Union's FP7 under REA Grant agreements no. PIOF-GA-2011-302520 GRAND-CRU and PIEF-GA2012-330731 Dense4Green, from the European Commission in the framework of the FP7 Network of Excellence in Wireless COMmunications NEWCOM\# (Grant agreement no. 318306), and from the French National Research Agency (ANR) research grant NETLEARN (contract no. ANR-13-INFR-004).
Motivated by all this, in this paper, we analyze the uplink component of an orthogonal frequency-division multipleaccess (OFDMA)-based small cell network. In particular, we propose a game-theoretic framework to examine the optimal power allocation over the available sub-carriers at each transmitter, designed to maximize each link's individual energyaware utility. Specifically, the utility of each transmitter is defined as the achieved throughput per unit of power, accounting for both the power required for data transmission and that required by the circuit components of the wireless device (such as amplifiers, mixer, oscillator, and filters) [2][4]. In addition to the above, if the users also have a minimum rate requirement that must be achieved, the resulting game departs from the classical framework put forth by Nash [5], and becomes a Debreu-type game in which the actions available to each user depend on the power profile of other users [6]. In this setting, the relevant solution concept is that of a Debreu equilibrium (which is also commonly known as a generalized Nash equilibrium); the existence of this state is then proved by suitably extending the results of [6] to a setting with non-compact action sets, and its structure is characterized using techniques from fractional programming [7]. As we shall see, Debreu equilibria correspond to the fixed points of a water-filling best response operator where the water level is a function of the minimum rate constraints and each user's power envelope [4]. The theoretical analysis is then adopted to derive an iterative and distributed power allocation algorithm whose convergence and performance are assessed by means of numerical results.

The most relevant works in the above context are [8] and [9]. In [8], the authors examine a similar distributed energy-aware resource allocation problem but the users are not assumed to have any hard minimum rate requirements, so the resulting non-cooperative game is a classical Nash game with continuous action sets. The added quality-of-service (QoS) requirement complicates the analysis of the game considerably: to prove the equilibrium existence, one must go beyond standard Nash equilibrium results, since a user's admissible power allocation profile depends on the power profile of all other users; also, the question of the uniqueness of an equilibrium, in our case, seems to be a very difficult issue, which is left as a future work. Much closer in spirit is the very recent companion paper [9], whose system model is essentially equivalent to the one that we study in this paper (including the minimum QoS constraint at the user level). That said, by taking an approach 
based on fractional programming, we are able to chart out here the convergence properties of the proposed water-filling best response algorithm, and we are also able to provide a rigorous proof of the existence of a Debreu equilibrium by extending the results of [6] to a non-compact setting (by contrast, in [9] this result is announced but not proven).

Paper outline: In Sect. II, we introduce the small-cell network model, which is then formulated as a Nash/Debreu energy-aware power allocation game in Sect. III (where we prove the existence of a Debreu equilibrium and characterize its structure). In Sect. IV, we propose a distributed algorithm, which allows the users to converge to the game's equilibrium in a distributed fashion. The convergence of the proposed distributed algorithm and the energy-efficiency of the algorithm's end state are then evaluated via numerical simulations in Sect. V.

Notational conventions: Matrices and vectors are denoted by bold letters, $\mathbf{I}_{L}, \mathbf{0}_{L}$, and $\mathbf{1}_{L}$ are the $L \times L$ identity matrix, the $L \times 1$ all-zero column vector, and the $L \times 1$ allone column vector, respectively, and $\|\cdot\|^{2},(\cdot)^{T}$ and $(\cdot)^{H}$ denote Euclidean norm of the enclosed vector, transposition and Hermitian transposition respectively. The notation $(x)^{+}$ stands for $\max (0, x)$ whereas $W(\cdot)$ denotes the Lambert $W$ function [10], defined to be the multivalued inverse of the function $z=W(z) e^{W(z)}$ for any $z \in \mathbb{C}$. Finally, if $\mathcal{A}_{k}$, $k=1, \ldots, K$ is a finite family of sets, and $a_{k} \in \mathcal{A}_{k}$, we will use the notation $\left(a_{k} ; \mathbf{a}_{-k}\right) \in \prod_{k} \mathcal{A}_{k}$ as shorthand for the profile $\left(a_{1}, \ldots, a_{k}, \ldots, a_{K}\right)$.

\section{SYSTEM MODEL AND PROBLEM FORMULATION}

We consider the uplink of a network composed by $S$ small cells operating in an OFDMA-based open-access licensed spectrum. The $s$ th small cell uses a set of orthogonal subcarriers to serve the $K_{s}$ user equipments (UEs) falling within its coverage radius $\rho_{s}$. For simplicity, we assume that the same set of subcarriers $\mathcal{N}=\{1, \ldots, N\}$ is used by all small cells. The latter is assigned by the macrocell network and does not represent a parameter of our problem. To exploit the frequency diversity, we assume that the subcarrier spacing is larger than the coherence bandwidth $B_{c}$ experienced by each user. Each small-cell access point (SAP) is equipped with $M$ receiving antennas while a single antenna is employed at the UEs to keep the complexity of the front-end limited.

Let $\mathbf{h}_{k j, n} \in \mathbb{C}^{M \times 1}$ denote the uplink channel vector whose entries $\left[\mathbf{h}_{k j, n}\right]_{m}$ represent the (frequency) channel gains over subcarrier $n$ from the $j$ th UE to the $m$ th receive antenna of user $k$ th serving SAP, i.e., the $s$ th SAP whose distance from user $k$ is smaller than $\rho_{s}$ with $k, j \in \mathcal{K}=\{1, \ldots, K\}$ and $K=$ $\sum_{s=1}^{S} K_{s}$. The vector $\mathbf{x}_{k, n} \in \mathbb{C}^{M \times 1}$ collecting the samples received at the UE $k$ 's serving SAP over the $n$th subcarrier can be written as

$$
\mathbf{x}_{k, n}=\sum_{j=1}^{K} \mathbf{h}_{k j, n} \sqrt{p_{j, n}} z_{j, n}+\mathbf{w}_{k, n}
$$

where $\mathbf{w}_{k, n} \in \mathbb{C}^{M \times 1}$ is a Gaussian vector with zero mean and covariance matrix $\sigma^{2} \mathbf{I}_{M}$ accounting for background noise, whereas $p_{j, n}$ and $z_{j, n}$ denote UE $j$ 's transmit power and data symbol over subcarrier $n$, respectively. To keep the complexity of the SAP at a tolerable level, a simple linear detection scheme is employed for data detection. This means that the entries of $\mathbf{x}_{k, n}$ are linearly combined to form $y_{k, n}=\mathbf{g}_{k, n}^{H} \mathbf{x}_{k, n}$, where $\mathbf{g}_{k, n}$ is the vector employed for recovering the data transmitted by user $k$ over subcarrier $n$. The signal-to-interference-plusnoise ratio (SINR) achieved by user $k$ at its serving SAP over subcarrier $n$ takes the form

$$
\gamma_{k, n}=\mu_{k, n}\left(\mathbf{p}_{-k, n}\right) p_{k, n}
$$

with

$$
\mu_{k, n}\left(\mathbf{p}_{-k, n}\right)=\frac{\left\|\mathbf{g}_{k, n}^{H} \mathbf{h}_{k k, n}\right\|^{2}}{\left\|\mathbf{g}_{k, n}\right\|^{2} \sigma^{2}+\sum_{j \neq k}\left\|\mathbf{g}_{k, n}^{H} \mathbf{h}_{k j, n}\right\|^{2} p_{j, n}}
$$

where we have explicitly reported the dependence on $\mathbf{p}_{-k, n}=$ $\left[p_{1, n}, \ldots, p_{k-1, n}, p_{k+1, n}, \ldots, p_{K, n}\right]^{T}$, which is the vector collecting all powers transmitted over subcarrier $n$ except user $k$ 's one. Using (2), the achievable rate (normalized to the subcarrier bandwidth, and thus measured in $\mathrm{b} / \mathrm{s} / \mathrm{Hz}$ ) of the $k$ th user is given by

$$
r_{k}(\mathbf{p})=\sum_{n=1}^{N} \log _{2}\left(1+\gamma_{k, n} / \Gamma\right)
$$

where $\Gamma$ is the SINR gap with respect to the Shannon capacity [11], and $\mathbf{p}=\left[\mathbf{p}_{1}^{T}, \ldots, \mathbf{p}_{K}^{T}\right]^{T} \in \mathbb{R}_{+}^{K \times N}$ collects the transmit powers by all users over all subcarriers, where the (row) vector $\mathbf{p}_{k}=\left[p_{k, 1}, \ldots, p_{k, N}\right]^{T}$ denotes user $k$ 's powers over all subcarriers, with $p_{k, n} \geq 0$ (if $p_{k, n}=0$, user $k$ is not transmitting over subcarrier $n$ ). Note that user $k$ 's multiple access interference (MAI), measured by the summation at the denominator of (3), comes from both intra-cell interference (generated by other UEs being served by the same SAP) and inter-cell interference (from UEs served by all other $S-1$ SAPs), whereas macro-cell users are assumed to be orthogonal thanks to a proper frequency resource planning operated by the macro-cell network (if needed, macro-cell interference can be included into $\sigma^{2}$ ). To simplify notations, the dependence of $\mu_{k, n}$ and $r_{k}$ is not made explicit from now on.

As mentioned in Sect. I, an energy-efficient design of the network, which is of primary importance when dealing with mobile, battery-power UEs, must properly take into account the energy consumption incurred by each UE. To this aim, it is worth noting that, beside the radiative powers $\mathbf{p}_{k}$ at the output of the radio-frequency front-end, each terminal $k$ also incurs circuit power consumption during transmission, mostly due to the power dissipated in the power amplifier [2], [4]. The overall power consumption $P_{T, k}$ of the $k$ th UE is thus given by

$$
P_{T, k}=p_{c}+P_{k}=p_{c}+\sum_{n=1}^{N} p_{k, n}
$$

where $\sum_{n=1}^{N} p_{k, n}=\mathbf{p}_{k}^{T} \mathbf{1}_{N}$ is the radiative power consumed by user $k$ over the whole spectrum, and $p_{c}$ represents the average current power consumed by the device electronics, which is assumed to be independent of the transmission state and equal for all UEs. Following [4], [12], the energy efficiency of the link can be measured (in $\mathrm{b} / \mathrm{J} / \mathrm{Hz}$ ) by the utility function

$$
u_{k}(\mathbf{p})=\frac{r_{k}}{P_{T, k}}=\frac{\sum_{n=1}^{N} \log _{2}\left(1+\mu_{k, n} p_{k, n} / \Gamma\right)}{p_{c}+\sum_{n=1}^{N} p_{k, n}}
$$


where the dependence of all others' transmit powers over all subcarriers is collected by the gains $\left\{\mu_{k, n}\right\}_{n=1}^{N}$. Observe that, in data-oriented wireless networks, users are usually required to satisfy QoS requirements in terms of minimum achieved rates $\theta_{k} \geq 0$, i.e., $r_{k} \geq \theta_{k}$.

To summarize, the design of an energy-efficient resource allocation scheme, that encompasses both subcarrier allocation and power control (by setting, for each UE $k, p_{k, n}=0$ on unused subcarriers, and $p_{k, n}>0$ on used subcarriers), requires to solve, for each UE $k$, the following optimization problem:

$$
\begin{aligned}
\mathbf{p}_{k}^{\star}=\underset{\mathbf{p}_{k} \in \mathbb{R}_{+}^{N}}{\arg \max } & \frac{\sum_{n=1}^{N} \log _{2}\left(1+\mu_{k, n} p_{k, n} / \Gamma\right)}{p_{c}+\sum_{n=1}^{N} p_{k, n}} \\
\text { subject to } & p_{k, n} \geq 0 \quad \forall n=1, \ldots, N \\
& \sum_{n=1}^{N} \log _{2}\left(1+\mu_{k, n} p_{k, n} / \Gamma\right) \geq \theta_{k}
\end{aligned}
$$

where the constraint (8) ensures each transmit power to be positive, whereas (9) forces each user to fulfill a requirement on the minimum normalized rate $\theta_{k}$. Note that, unlike other formulations in the field of OFDMA resource allocation (see for example [13], [14]), here the subcarrier selection and power loading problems are tackled in a joint manner. Furthermore, the interplay among the UEs in $\mathcal{K}$ makes (7) a multidimensional optimization problem in which each UE $k \in \mathcal{K}$ aims at unilaterally choosing its own transmit power allocations $\mathbf{p}_{k}$ so as to optimize its own link energy efficiency $u_{k}(\mathbf{p})$. In doing this, each UE affect the choice of all other UEs as well.

\section{GAME-THEORETIC RESOURCE ALLOCATION}

A natural framework for studying the strategic inter-user interactions that arise from the system model of the previous section is offered by the theory of non-cooperative games with continuous (and action-dependent) action sets. Following Debreu [6] (see also [15]), we will thus consider a noncooperative game $\mathfrak{G} \equiv \mathfrak{G}(\mathcal{K}, \mathcal{P}, u)$ defined as follows:

a) The players of $\mathfrak{G}$ will comprise the set $\mathcal{K}$ of UEs.

$b)$ The total action set of player $k$ representing all transmit power profiles (including possibly unfeasible ones) will be:

$$
\mathcal{P}_{k}^{\mathrm{nc}}=\left\{\mathbf{p}_{k} \in \mathbb{R}^{N}: p_{k, n} \geq 0 \text { for all } n=1, \ldots, N\right\} \text {. }
$$

Otherwise, in the presence of the rate constraints (9), the feasible action set of player $k$ given a power allocation profile $\mathbf{p}_{-k} \in \mathcal{P}_{-k}^{\text {nc }} \equiv \prod_{\ell \neq k} \mathcal{P}_{\ell}^{\text {nc }}$ of other users will be:

$$
\mathcal{P}_{k}\left(\mathbf{p}_{-k}\right)=\left\{\mathbf{p}_{k} \in \mathcal{P}_{k}^{\mathrm{nc}}: r_{k}(\mathbf{p}) \geq \theta_{k}\right\} .
$$

c) The utility function of player $k$ will be given by (6).

A first question that arises is whether this game is feasible in the sense that there exists a power allocation profile $\mathbf{p}=$ $\left(\mathbf{p}_{1}, \ldots, \mathbf{p}_{K}\right) \in \prod_{k} \mathcal{P}_{k}^{\text {nc }}$ such that

$$
\mathbf{p}_{k} \in \mathcal{P}_{k}\left(\mathbf{p}_{-k}\right) \text { for all } k \in \mathcal{K} \text {. }
$$

The feasibility of the game depends non-trivially on the users' channels and rate constraints [9], and it is easy to construct examples where there are no feasible profiles with finite power. ${ }^{1}$ Albeit important in its own right, this feasibility

\footnotetext{
${ }^{1}$ From a mathematical viewpoint, a compactification argument can be used to show that the game always admits a feasible point if infinite powers and finite rates are allowed, but, of course, this has little practical relevance.
}

question will not be addressed here; instead, assuming that the problem is feasible, we will focus on power allocation profiles that are unilaterally stable for all users - and thus provide a sense of system-wide stability as well.

In this framework, the most widely used solution concept is a generalization of the notion of Nash equilibrium, known as Debreu equilibrium [6] (and sometimes also referred to as a generalized Nash equilibrium [15]). Formally:

Definition 1: A transmit power profile $\mathbf{p}^{\star}$ is a Debreu equilibrium of $\mathfrak{G}(\mathcal{K}, \mathcal{P}, u)$ if, for all players $k \in \mathcal{K}$, we have $\mathbf{p}_{k}^{\star} \in \mathcal{P}_{k}\left(\mathbf{p}_{-k}^{\star}\right)$ and

$$
u_{k}\left(\mathbf{p}^{\star}\right) \geq u_{k}\left(\mathbf{p}_{k} ; \mathbf{p}_{-k}^{\star}\right)
$$

for all $\mathbf{p}_{k} \in \mathcal{P}_{k}\left(\mathbf{p}_{-k}^{\star}\right)$.

Debreu equilibria are of particular interest in the context of distributed systems because they offer a stable solution of the game in which every player (in this case, the small-cell UE) is satisfied with its action choices and does not wish to deviate from (and thus destabilize the system) if other players stick to their chosen actions. Accordingly, in the rest of this section, we will treat the problem of equilibrium existence in the power allocation game $\mathfrak{G}$, leaving the question of convergence to such state to Sect. IV.

Debreu's original paper [6] provides a general existence result for games of this kind under the assumptions that:

1) the players' constrained action sets $\mathcal{P}_{k}\left(\mathbf{p}_{-k}\right)$ are compact, convex and nonempty for all $\mathbf{p}_{-k} \in \mathcal{P}_{-k}$;

2) $\mathcal{P}_{k}\left(\mathbf{p}_{-k}\right)$ varies continuously with $\mathbf{p}_{-k}$ (in the sense that the graph of the set-valued correspondence $\mathbf{p}_{-k} \mapsto$ $\mathcal{P}_{k}\left(\mathbf{p}_{-k}\right)$ is closed); and

3) each player's utility function is quasi-concave over the player's individual actions.

In our setting, the Shannon rate function $r_{k}\left(\mathbf{p}_{k} ; \mathbf{p}_{-k}\right)$ (4) is concave in $\mathbf{p}_{k}$ and unbounded from above, so $\mathcal{P}_{k}\left(\mathbf{p}_{-k}\right)$ is convex and nonempty for all $\mathbf{p}_{-k} \in \mathcal{P}_{-k}^{\text {nc }}$; moreover, $\mathcal{P}_{k}\left(\mathbf{p}_{-k}\right)$ varies continuously with $\mathbf{p}_{-k}$ since constraints (9) are themselves continuous in $\mathbf{p}_{-k}$. Finally, it can be easily shown that $u_{k}\left(\mathbf{p}_{k} ; \mathbf{p}_{-k}\right)$ is quasi-concave in $\mathbf{p}_{k}$ since, for any $\xi \in \mathbb{R}, u_{k}\left(\mathbf{p}_{k} ; \mathbf{p}_{-k}\right) \geq \xi$ if and only if

$$
r_{k}\left(\mathbf{p}_{k} ; \mathbf{p}_{-k}\right)-\xi\left(p_{c}+\sum_{n=1}^{N} p_{k, n}\right) \geq 0
$$

and since the set defined by this inequality is convex for every $\mathbf{p}_{-k} \in \mathcal{P}_{-k}$ (recall that $r_{k}\left(\mathbf{p}_{k} ; \mathbf{p}_{-k}\right)$ is concave in $\left.\mathbf{p}_{k}\right)$, our claim follows.

Unfortunately however, the sets $\mathcal{P}_{k}\left(\mathbf{p}_{-k}\right)$ are not compact, so Debreu's equilibrium existence result does not apply. Nonetheless, an extension of the reasoning of [6] leads to the following result (see Appendix A for the proof).

Proposition 1: If the game $\mathfrak{G} \equiv \mathfrak{G}(\mathcal{K}, \mathcal{P}, u)$ is feasible, then it admits a Debreu equilibrium. 
Having established the existence of a Debreu equilibrium, we now turn to investigate its structure. Denoting by

$$
\begin{aligned}
& \alpha_{k}=\frac{1}{N}\left(p_{c}-\sum_{n=1}^{N} \Gamma / \mu_{k, n}\right) \\
& \beta_{k}=\frac{1}{N} \sum_{n=1}^{N} \ln \left(\mu_{k, n} / \Gamma\right)
\end{aligned}
$$

the following result is proved in Appendix B using the fractional programming paradigm.

Proposition 2: At the Debreu equilibrium of $\mathfrak{G}$, the elements $p_{k, n}^{\star}$ of the optimal transmit power profile $\mathbf{p}^{\star}$ are the solutions to the following fixed-point system of equations:

$$
p_{k, n}^{\star}=\left(\frac{1}{\lambda_{k}^{\star}}-\frac{\Gamma}{\mu_{k, n}}\right)^{+}
$$

where

$$
\lambda_{k}^{\star}=\min \left(\lambda_{k}, \bar{\lambda}_{k}\right)
$$

with

$$
\lambda_{k}=e^{\left(\beta_{k}-1\right)-W\left(\alpha_{k} \cdot e^{\beta_{k}-1}\right)}
$$

being the water level of the water-filling operator (17) when the problem (7) is solved without the minimum-rate constraints (9) (or equivalently, $\theta_{k}=0 \forall k$ ), where $W(\cdot)$ denotes the Lambert $W$ function [10], and ${ }^{2}$

$$
\bar{\lambda}_{k}=\frac{1}{\Gamma} \sqrt[N]{\frac{1}{2^{\theta_{k}}} \prod_{n=1}^{N} \mu_{k, n}}
$$

is the water level of (17) when all minimum-rate constraints (9) are simultaneously met with equality (i.e., (7) reduces to a power minimization given rate constraints $r_{k}=\theta_{k}$ ).

\section{DistRIBUTED IMPLEMENTATION}

To derive a practical criterion to let each small-cell UE $k \in \mathcal{K}$ reach the Debreu equilibrium of $\mathcal{G}$ in a distributed fashion, we start by assuming that the UEs with indices $j \neq k$ have already chosen their optimal transmit powers (i.e., in an asynchronous resource allocation scenario). This amounts to assuming $\mathbf{p}_{-k}=\mathbf{p}_{-k}^{\star}$. Hence, from (3), we have that the gains $\mu_{k, n}\left(\mathbf{p}_{-k, n}^{\star}\right)$ needed to implement (17) can be obtained by

$$
\mu_{k, n}\left(\mathbf{p}_{-k, n}^{\star}\right)=\frac{\gamma_{k, n}}{p_{k, n}}
$$

for all $n \in \mathcal{N}$. This means that the only information that is not locally available at the $k$ th UE to compute the optimal powers $\left\{p_{k, n}^{\star}\right\}$ is the set of SINRs $\left\{\gamma_{k, n}\right\}$ measured at UE $k$ 's serving SAP, that can be fed back with a modest feedback rate requirement on the return channel (a discussion on the impact of a limited feedback can be adapted to this specific scenario from [16]).

Based on the above considerations, we can derive an iterative and fully decentralized algorithm to be adopted by each UE $k$ at each time step $t$ to solve the fixed-point

\footnotetext{
${ }^{2}$ The closed-form expressions (19) and (20) apply only when all subcarriers $n$ are active, i.e., $p_{k, n}>0$. Please refer to Sect. IV for a practical method to compute $\lambda_{k}$ and $\bar{\lambda}_{k}$ in the general case $p_{k, n} \geq 0$.
}
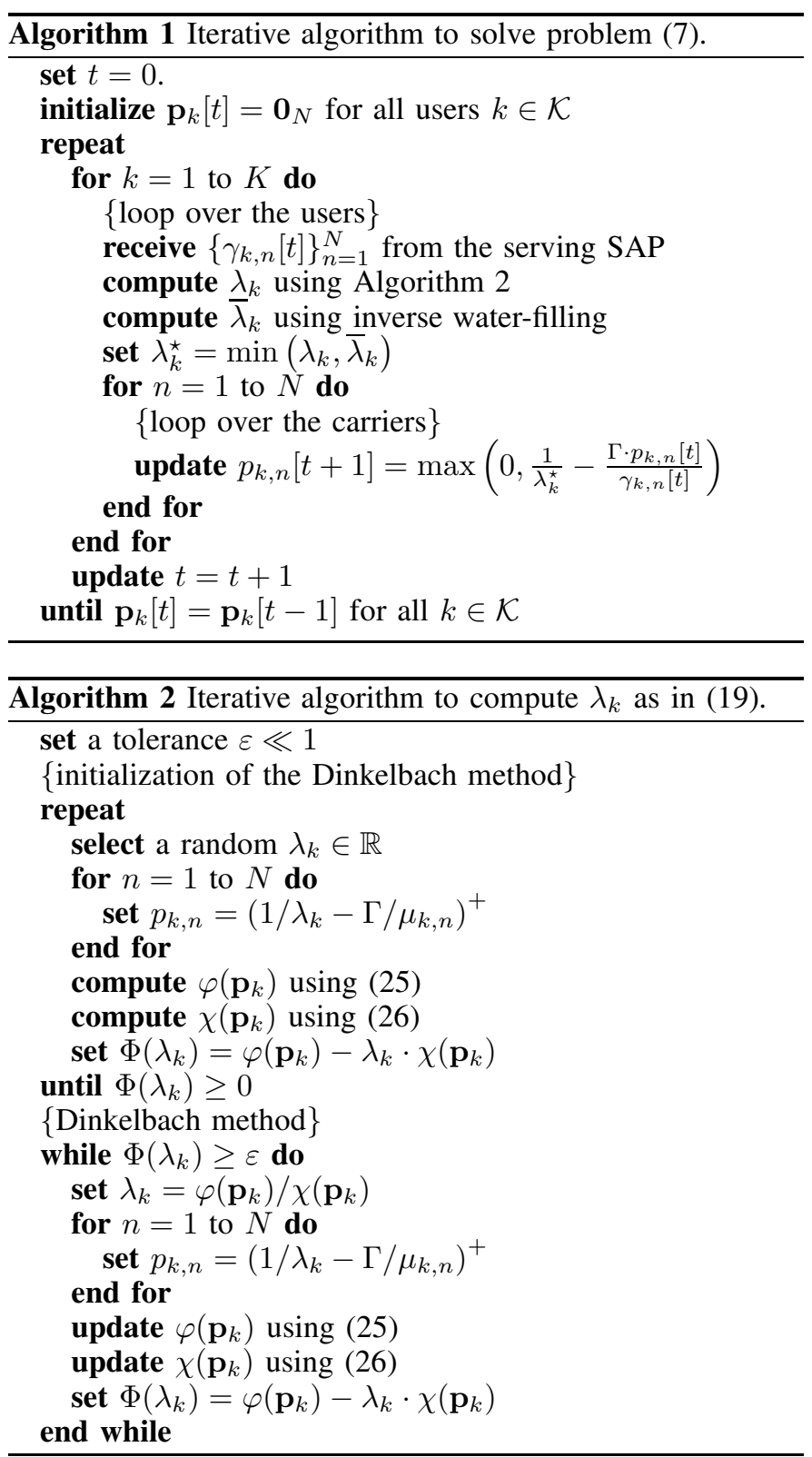

system of equations (17) with a low-complexity, scalable and adaptive procedure. The pseudo-code for the whole network is summarized in Algorithm 1. Note that, in practice, each UE $k$ needs only to implement the steps enclosed in the inner cycle, and this algorithm is thus suitable for a dynamic network configuration, since each UE only requires the SINRs fed back by the serving SAP, without any further information on the network and/or small-cell status.

For the sake of clarity, the algorithm to compute $\lambda_{k}$ as in (19) is reported in Algorithm 2, whereas $\bar{\lambda}_{k}$ can easily be computed using standard water-filling algorithms (e.g., see [7]). Note that, although (19) is derived analytically in a closed form, it is attractive to use the iterative method outlined in Algorithm 2, that takes advantage of the Dinkelbach method [17], a numerical method based on the application of Newton's method that significantly reduces the computational complexity compared to the evaluation of the Lambert $W$ function. For the sake of brevity, Algorithm 2 makes use 


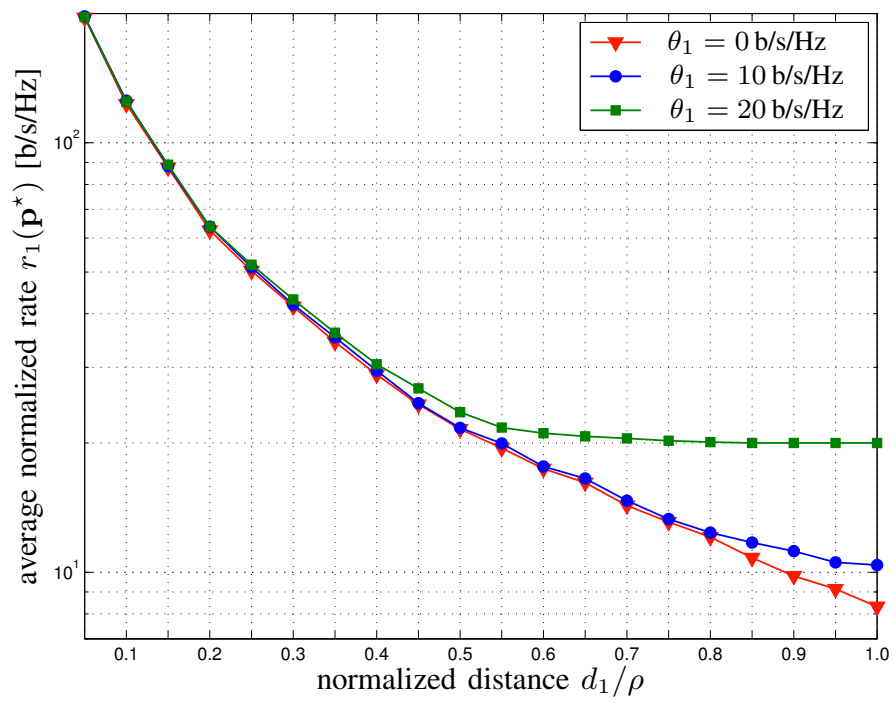

Fig. 1. Average normalized rate at the equilibrium as a function of the normalized distance from the SAP.

of some functions introduced in Appendix B. Throughout the simulations reported in Sect. $\mathrm{V}$, the tolerance is set to $\varepsilon=10^{-5}$.

The convergence of Algorithm 1 to the equilibrium point is assessed numerically in the next section by means of extensive simulations. A formal proof of the convergence is beyond the scope of this work, and it is currently under investigation. A possible solution in this direction comes from the observation that the power allocation $p_{k, n}[t+1]$ in Algorithm 1 is updated according to a water-filling strategy, in which the water level $\lambda_{k}^{\star}$ is computed as $\lambda_{k}^{\star}=\min \left(\lambda_{k}, \bar{\lambda}_{k}\right)$. Studying the contraction properties of this water-filling operator is likely to represent the right way to prove the convergence towards the equilibrium (e.g., see [18] for more details).

\section{Simulation Results}

In this section, we propose some numerical results to evaluate the performance of the proposed algorithm for different working conditions. Throughout the simulations, we adopt the following parameters (see [2] and references therein). We consider a $200 \mathrm{~m} \times 200 \mathrm{~m}$ area populated by $S=8$ randomly distributed small cells, each having a radius $\rho_{s}=\rho=20 \mathrm{~m}$ and a forbidden area with radius $0.2 \mathrm{~m}$. The set of available subcarriers is composed by $N=32$ subcarriers, each having a bandwidth $B \approx 11 \mathrm{kHz}$ and spaced by $350 \mathrm{kHz}$, whereas each UEs coherence bandwidth is assumed to be $B_{c} \approx 90 \mathrm{kHz}$, using a 24-tap channel model to reproduce multipath effects. To include the effects of fading and shadowing into our model, we use a path-loss exponent equal to $\varsigma=4$. For simplicity, we assume perfect channel estimation at the receiver side, and we consider the maximum ratio combining (MRC) technique, which amounts to setting $\mathbf{g}_{k, n}=\mathbf{h}_{k k, n}$ for all $k \in \mathcal{K}$ and $n \in \mathcal{N}$. We also assume an SINR gap equal to $\Gamma=1=0 \mathrm{~dB}$ and an AWGN per-subcarrier power equal to $\sigma^{2}=0.137 \mathrm{fW}$. Without loss of generality, we will measure the performance for a specific user (say user 1) within a small cell, by averaging over all possible positions of the users, uniformly randomizing their minimum-rate constraints $\theta_{k}$ in $[0,20][\mathrm{b} / \mathrm{s} / \mathrm{Hz}]$ for $k \neq 1$.

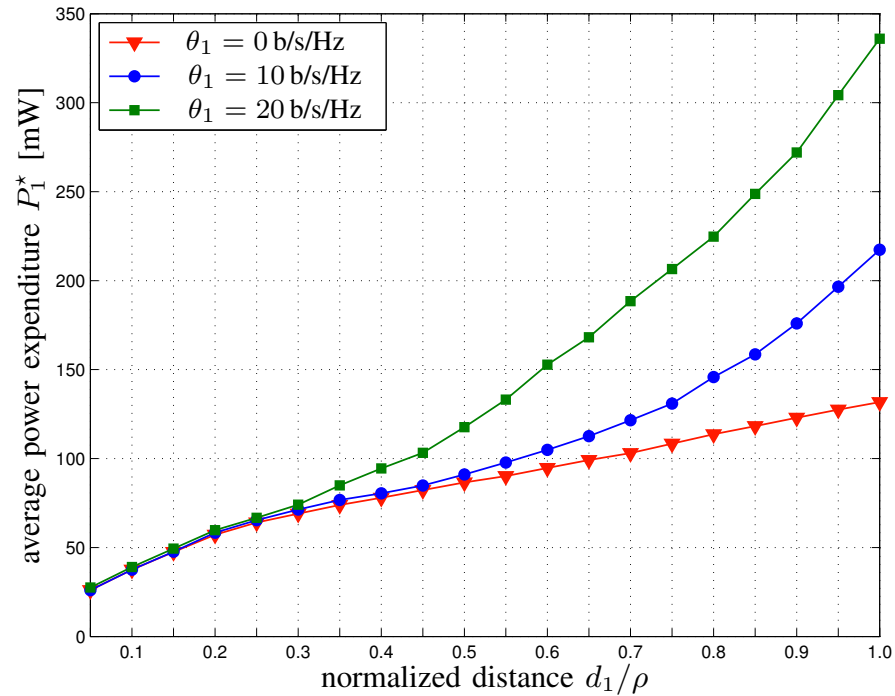

Fig. 2. Average transmit power at the equilibrium as a function of the normalized distance from the SAP.

Unless otherwise specified, we assume each $\operatorname{SAP} s$ to be equipped with $M=2$ received antennas, the number of users per small cell to be $K_{s}=8$, the nonradiative power to be $p_{c}=100 \mathrm{~mW}$, and the rate constraint for the user of interest to be $\theta_{1}=10 \mathrm{~b} / \mathrm{s} / \mathrm{Hz}$.

Figs. 1 and 2 depict the average total transmit powers and the achievable (normalized) rates at the equilibrium as functions of the (normalized) distance between the observed user and its SAP, respectively, averaged over 10,000 independent network realizations. Red lines represent the case without rate constraints $\theta_{1}=0 \mathrm{~b} / \mathrm{s} / \mathrm{Hz}$, whereas blue and green lines report the cases $\theta_{1}=10 \mathrm{~b} / \mathrm{s} / \mathrm{Hz}$ and $\theta_{1}=20 \mathrm{~b} / \mathrm{s} / \mathrm{Hz}$, respectively. As can be seen, when the UE is close to the serving SAP $d_{1} / \rho \leq 0.3$, the equilibrium point in the three cases is exactly the same, as the energy-efficient formulation lets the user achieve an equilibrium rate which is significantly larger than the minimum one. When on the contrary the reference user is close to the cell edge (say $d_{1} / \rho \geq 0.8$ ), the MAI becomes significant, and thus the equilibrium rate given by the energy efficient formulation approaches the minimum one. This is particularly apparent in Fig. 1, and is reflected in the average total power consumption reported in Fig. 2. This behavior, although dependent from the particular network settings, is true in general. Note that the critical normalized distance, at which $r_{1}\left(\mathbf{p}^{\star}\right) \approx \theta_{1}$, decreases as either the constraint $\theta_{1}$ increases, or the degrees of freedom $N \cdot M$, that is directly connected to the number of available resources in the network, decreases with respect to the number of users $K=\sum_{s} N_{s}=64 .^{3}$ Interestingly, as confirmed by numerical results not reported here for the sake of brevity, which are also in line with the simulations reported in [9], the energy efficiency (in terms of the utility (6) achieved at the equilibrium) is weakly dependent on the constraint $\theta_{1}$ : although it decreases as $\theta_{1}$ increases, the variation is negligible, thus introducing fairness into the network.

${ }^{3}$ A rule of thumb to properly drive the network is to have $N \cdot M \geq K$. In this case, we have on purpose reported a borderline scenario, in which $N=32, M=2$, and $K=64=N \cdot M$. 


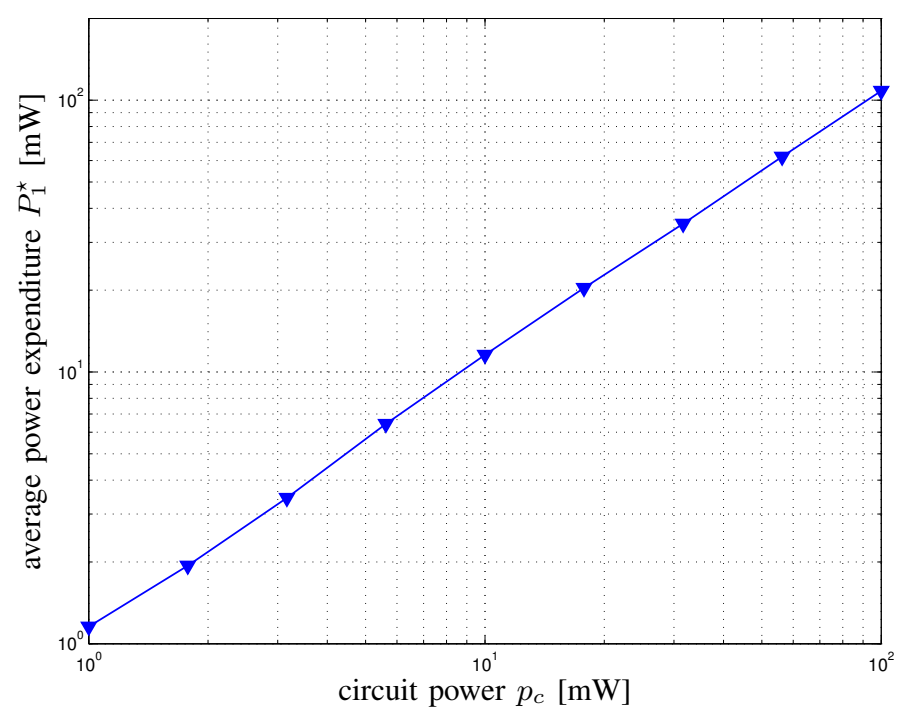

Fig. 3. Average transmit power at the equilibrium as a function of the circuit power.

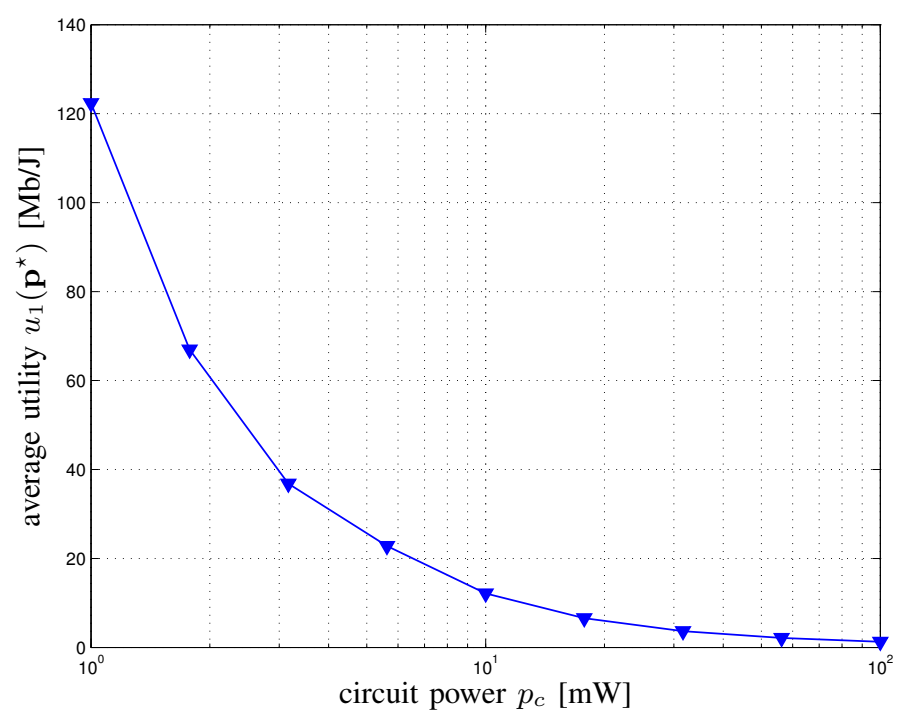

Fig. 4. Average utility at the equilibrium as a function of the circuit power.

To evaluate the impact of the circuit power $p_{c}$ on the energy efficiency of the system, in Figs. 3 and 4 we report the performance of the proposed algorithm as a function of $p_{c}$, averaged over 100, 000 independent network realizations. Note that, for all selected nonradiative powers $p_{c} \in[1,100] \mathrm{mW}$, the hypothesis $p_{c} \gg \sigma^{2}$ holds, which is in line with the state of the art for radio-frequency and baseband transceiver modeling [2]. As can be seen in Fig. 3, the total power consumption at the equilibrium $P_{1}\left(\mathbf{p}^{\star}\right)$ is directly proportional to $p_{c}$. Otherwise stated, the energy-efficient equilibrium point is highly impacted by the nonradiative power, and bit-per-Joule metric tells us to use a radiative power which is comparable with the nonradiative one. Interestingly, the (normalized) achievable rates at the equilibrium point are $r_{1}\left(\mathbf{p}^{\star}\right) \approx 20 \mathrm{~b} / \mathrm{s} / \mathrm{Hz}$ irrespectively of $p_{c}$. This justifies the behavior of the achieved utilities at the equilibrium, reported in Fig. 4, and confirms a result which is well-known in the literature (e.g., see [4], [19]):

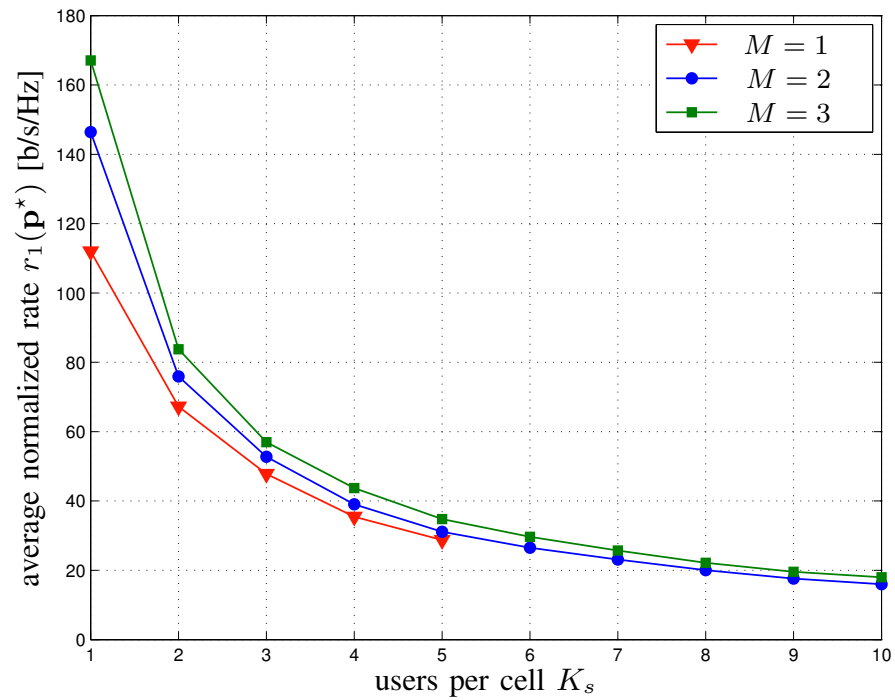

Fig. 5. Average normalized rate at the equilibrium as a function of the number of users per small cell.

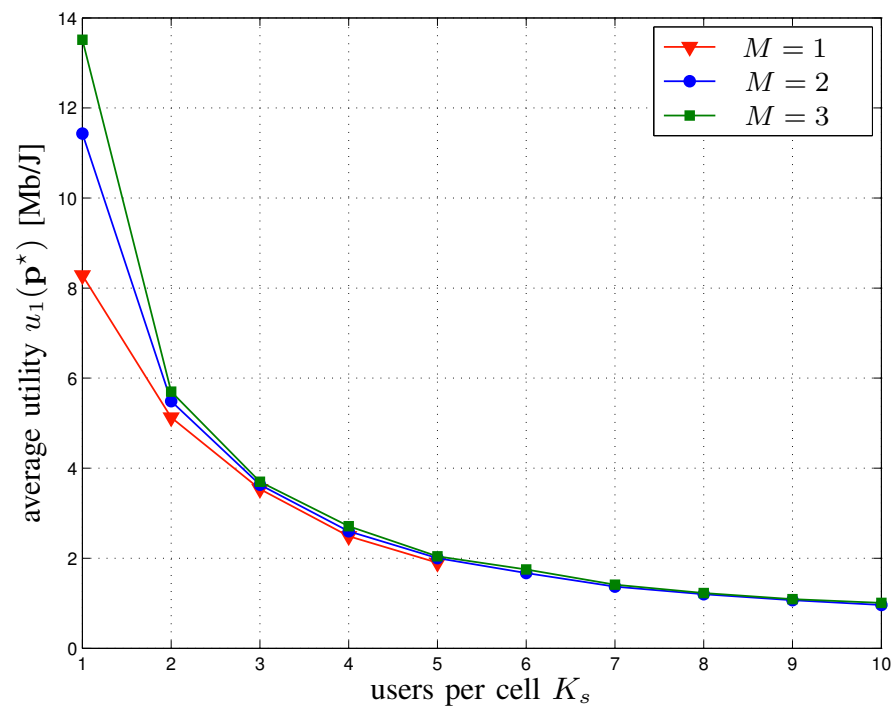

Fig. 6. Average utility at the equilibrium as a function of the number of users per small cell.

the energy efficiency increases as the circuit (nonradiative) power decreases. Hence, reducing $p_{c}$ can achieve a two-fold goal, thus further boosting the research in this field: not only is it expedient to reduce the constant power consumption (from an electronics point of view), but also it leads energyaware terminals to reduce their radiative power when they aim at maximizing their bit-per-Joule performance (from an information-theoretic and resource-allocation perspective).

Finally, Figs. 5 and 6 report the performance of the proposed resource allocation scheme as a function of the number of users per cell $K_{s}$ for three different receive architectures: $M=1$ (red lines), $M=2$ (blue lines), and $M=3$ (green lines) antennas, respectively. As can easily be guessed, the degrees of freedom $N \cdot M$ impact on the occurrence of feasible scenarios: as confirmed by simulations, when the total number 
of users $K=8 N_{s}$ is larger than $N \cdot M=32 M$, the resources available in the network are scarce to accommodate all the rate requests $\theta_{k}$. To provide significant performance results, we plot only network configurations that yield feasible scenarios with an occurrence larger than $70 \%$ of the times. This is the reason why only $K_{s} \leq 5$ is reported for the case $M=1$, whereas $M=\{2,3\}$ are able to accommodate a larger population of users without impacting on the required minimum performance $\theta_{k}$. As can be seen in Fig. 5, increasing the number of users $K_{s}$ increases the contribution from both intra-cell and inter-cell MAI, which in turn decreases the average normalized achievable rate at the equilibrium. However, note that increasing $M$ yields a larger $r_{1}\left(\mathbf{p}^{\star}\right)$, since the SAP can better separate the users due to a larger space diversity given by the singleinput-multiple-output (SIMO) configuration (and thus a larger number of degrees of freedom).

This behavior is confirmed by Fig. 6, that measured the utility (6) as a function of the same parameters. As expected, in all network configurations increasing $K_{s}$ decreases $u_{1}\left(\mathbf{p}^{\star}\right)$, due to increasing the MAI. However, note that the difference is not significant for all receiver configurations. This means that, if the only concern is the energy efficiency of the system, then increasing the number of antennas does not yield significant advantages. On the contrary, if also the spectral efficiency is critical (i.e., if larger $\theta_{k}$ 's are needed), then increasing $M$ is a viable solution to achieve a proper resource allocation, as witnessed by larger achievable rates at the equilibrium (see Fig. 5). It is worth noting that these considerations hold true due to adopting a MRC technique, which is suboptimal for a proper MAI management. Other schemes, such as zero-forcing (ZF) or minimum mean-square error (MMSE) approaches, which are out-of-scope of the present contribution, might lead to exploiting better the spatial diversity of the MISO configurations, and thus larger differences in the achieved energy efficiency.

\section{CONCLUSIONS AND PERSPECTIVES}

In this paper, we proposed a distributed power allocation scheme for energy-aware, non-cooperative wireless users with minimum rate constraints in an uplink multicarrier smallcell network. By modeling this scenario as a non-cooperative game in the sense of Debreu, we proved the existence of an equilibrium state and we characterized it as the fixed point of a water-filling operator using techniques borrowed from fractional programming. To attain this equilibrium in a distributed fashion, we also proposed an iterative solution method based on an iterative water-filling best response process, whose convergence and performance was assessed by numerical simulations. Performance results show that reducing the nonradiative power consumed by the user device electronics is particularly critical to improve the performance of mobile terminals in terms of energy efficiency, which is also highly impacted by the receiver configuration.

Further work is needed: $i$ ) to assess the feasibility of the problem given a particular network realization; ii) to analytically prove the convergence of the iterative algorithm to the equilibrium point; iii) to assess its complexity as a function of the system parameters; and $i v$ ) to evaluate the impact of different receiver architectures on the spectral and energy efficiency of the network.

\section{APPENDIX A \\ PROOF OF PROPOSITION 1}

Fix some power vector $\mathbf{p}_{k} \in \mathcal{P}_{-k}^{\text {nc }}$, and let $\mathcal{P}_{k}^{\star}\left(\mathbf{p}_{-k}\right) \equiv$ arg $\max \left\{u_{k}\left(\mathbf{p}_{k} ; \mathbf{p}_{-k}\right): \mathbf{p}_{k} \in \mathcal{P}_{k}\left(\mathbf{p}_{-k}\right)\right\}$ be the best response set of player $k$ to the power profile $\mathbf{p}_{-k}$ of the other players. By the quasi-concavity of $u_{k}$ and Lemma 1 below, it follows that $\mathcal{P}_{k}^{\star}$ is compact; as a result, letting $M_{k}\left(\mathbf{p}_{-k}\right)$ denote the maximum value of $u_{k}$ over $\mathcal{P}_{k}\left(\mathbf{p}_{-k}\right)$, it follows that the set

$$
\mathcal{P}_{k}^{\prime}\left(\mathbf{p}_{-k}\right) \equiv\left\{\mathbf{p}_{k} \in \mathcal{P}_{k}^{\mathrm{nc}}: u_{k}\left(\mathbf{p}_{k} ; \mathbf{p}_{-k}\right) \geq(1-\varepsilon) M_{k}\left(\mathbf{p}_{-k}\right)\right\}
$$

will also be compact if $\varepsilon$ is chosen sufficiently small.

Define now the restricted game $\mathfrak{G}^{\prime} \equiv \mathfrak{G}^{\prime}\left(\mathcal{K}, \mathcal{P}^{\prime}, u\right)$ with the same data as $\mathfrak{G}$ except for the fact that the players' constrained action sets are now given by (22). Seeing as the sets $\mathcal{P}_{k}^{\prime}\left(\mathbf{p}_{-k}\right)$ are compact (by construction) and vary continuously with $\mathbf{p}_{-k}$ (simply note that all the functions involved in the definition of $\mathcal{P}_{k}^{\prime}\left(\mathbf{p}_{-k}\right)$ are themselves continuous $),{ }^{4} \mathfrak{G}^{\prime}$ will admit a Debreu equilibrium by Theorem 1 in [6]. On the other hand, given that the best response set $\mathcal{P}_{k}^{\star}\left(\mathbf{p}_{-k}\right)$ of player $k$ against $\mathbf{p}_{-k}$ in $\mathfrak{G}$ is contained in $\mathcal{P}_{k}^{\prime}\left(\mathbf{p}_{-k}\right)$ for all $\mathbf{p}_{-k} \in \mathcal{P}_{-k}^{\text {nc }}$ and for all $k \in \mathcal{K}$, it follows that any equilibrium of the restricted game $\mathfrak{G}^{\prime}$ will also be an equilibrium of $\mathfrak{G}$, and our proof is complete.

Lemma 1: Let $a_{n}>0, n=1, \ldots, N$, be positive constants, and let $b>0$. Then, the solution set of the problem

$$
\begin{array}{ll}
\operatorname{maximize} & f(\mathbf{p})=\frac{\sum_{n=1}^{N} \log \left(1+a_{n} p_{n}\right)}{b+\sum_{n=1}^{n} p_{n}} \\
\text { subject to } & p_{n} \geq 0
\end{array}
$$

is compact.

Proof: If the maximum set $\Omega_{f}$ of $f$ is not compact, there will exist an unbounded sequence $\mathbf{p}_{m} \in \Omega_{f}$, so, by descending to a subsequence of $\mathbf{p}_{m}$ if necessary, we may assume that $p_{n, m} \rightarrow \infty$ for some $n$. However, since $\lim _{p \rightarrow \infty} \log (1+a p) / p=0$ for all $a>0$, this implies that the maximum value of $f$ will be 0 , a contradiction.

\section{APPENDIX B \\ PROOF OF PROPOSITION 2}

The constrained optimization (7) can be cast into the paradigm of fractional programs, which are nonlinear programs where the objective function is a ratio of two real-valued functions. In particular, (7) can be rewritten as

$$
\mathbf{p}_{k}^{\star}=\underset{\mathbf{p}_{k} \in \mathcal{P}_{k}\left(\mathbf{p}_{-k}\right)}{\arg \max } \frac{\varphi\left(\mathbf{p}_{k}\right)}{\chi\left(\mathbf{p}_{k}\right)}
$$

where $\mathcal{P}_{k}\left(\mathbf{p}_{-k}\right)$ is defined as in (11), and

$$
\begin{aligned}
& \varphi\left(\mathbf{p}_{k}\right)=\sum_{n=1}^{N} \ln \left(1+\mu_{k, n} p_{k, n} / \Gamma\right), \\
& \chi\left(\mathbf{p}_{k}\right)=p_{c}+\sum_{n=1}^{N} p_{k, n} .
\end{aligned}
$$

\footnotetext{
${ }^{4}$ Importantly, given that the arg max operator is not continuous, this continuity property might fail if we had taken $\mathcal{P}_{k}^{\prime}\left(\mathbf{p}_{-k}\right) \equiv \mathcal{P}_{k}^{\star}\left(\mathbf{p}_{-k}\right)$. By artificially enlarging the players' best-response set, continuity is guaranteed.
} 
Using [4, Sect. II.A] we can see that solving problem (24) is equivalent to finding the root of the following nonlinear function:

$$
\Phi\left(\lambda_{k}\right)=\max _{\mathbf{p}_{k} \in \mathcal{P}_{k}\left(\mathbf{p}_{-k}\right)} \varphi\left(\mathbf{p}_{k}\right)-\lambda_{k} \chi\left(\mathbf{p}_{k}\right)
$$

where $\lambda_{k} \in \mathbb{R}$. To compute the solution of (24), let us first use (25) - (26), but without the constraint (9), so that $\mathbf{p}_{k} \in \mathbb{R}_{+}^{N}$ (i.e., only nonnegative powers are considered). The stationarity condition given by

$$
\left.\frac{\partial \varphi\left(\mathbf{p}_{k}\right)}{\partial p_{k, n}}\right|_{p_{k, n}=p_{k, n}^{\star}}-\left.\lambda_{k} \frac{\partial \chi\left(\mathbf{p}_{k}\right)}{\partial p_{k, n}}\right|_{p_{k, n}=p_{k, n}^{\star}}=0
$$

for all $n=1, \ldots, N$ using (25) - (26) becomes

$$
\frac{\mu_{k, n} / \Gamma}{1+\mu_{k, n} p_{k, n}^{\star} / \Gamma}-\lambda_{k}=0 \quad n=1, \ldots, N .
$$

Hence, considering $p_{k, n}^{\star} \geq 0$, the optimal power allocation becomes the waterfilling criterion (17), in which the water level $\lambda_{k}^{\star}$ is replaced by $\lambda_{k}$. By plugging (29) back into (27), we can finally compute the optimal power level $\lambda_{k}$ :

$$
-\ln \lambda_{k}+\left(\beta_{k}-1\right)=\alpha_{k} \lambda_{k}
$$

where the functions $\alpha_{k}$ and $\beta_{k}$ are defined as in (15) and (16), respectively. To provide a better insight on (30), let us try to write it in a closed form. To this aim, let us define

$$
\nu_{k}=-\ln \lambda_{k}+\left(\beta_{k}-1\right)
$$

so that (30) can be rewritten as

$$
\nu_{k} \cdot e^{\nu_{k}}=\alpha_{k} \cdot e^{\beta_{k}-1} .
$$

Using the Lambert function $W(\cdot)$, and inverting (31), after straightforward manipulation we get $\lambda_{k}$ as in (19).

When introducing back the constraint (9), we are placing a lower bound on $\varphi\left(\mathbf{p}_{k}\right): \varphi\left(\mathbf{p}_{k}\right) \geq \theta_{k}$. Following [4], this is equivalent to placing an upper bound $\bar{\lambda}_{k}$ on $\lambda_{k}$, that comes out of the inverse waterfilling criterion that minimizes $\chi\left(\mathbf{p}_{k}\right)$ given $\varphi\left(\mathbf{p}_{k}\right)=\theta_{k}$, and is equal to (20). Hence, the solution to (7) is given by (17), with $\lambda_{k}^{\star}$ computed as in (18).

\section{REFERENCES}

[1] J. Hoydis, M. Kobayashi, and M. Debbah, "Green small-cell networks," IEEE Veh. Technol. Mag., vol. 6, no. 1, pp. 37 - 43, 2011.
[2] E. Björnson, L. Sanguinetti, J. Hoydis, and M. Debbah, "Designing multi-user MIMO for energy efficiency: When is massive MIMO the answer?" in Proc. IEEE Wireless Commun. and Networking Conf. (WCNC), Istanbul, Turkey, Apr. 2014. [Online]. Available: http://arxiv.org/abs/1310.3843

[3] G. Bacci, E. V. Belmega, and L. Sanguinetti, "Distributed energyefficient power optimization in cellular relay networks with minimum rate constraints," in Proc. IEEE Intl. Conf. Acoustics, Speech and Signal Process. (ICASSP), Florence, Italy, May 2014.

[4] C. Isheden, Z. Chong, E. Jorswieck, and G. Fettweis, "Framework for link-level energy efficiency optimization with informed transmitter," IEEE Trans. Wireless Commun., vol. 11, no. 8, pp. 2946-2957, Aug. 2012

[5] J. Nash, "Non-cooperative games," Annals of Mathematics, vol. 54, no. 2, pp. 286-295, 1951.

[6] G. Debreu, "A social equilibrium existence theorem," Proc. Natl. Acad. Sci. USA, vol. 38, no. 10, pp. 886-893, October 1952.

[7] S. Boyd and L. Vandenberghe, Eds., Convex Optimization. Cambridge, UK: Cambridge Univ. Press, 2004.

[8] G. Miao, N. Himayat, G. Li, and S. Talwar, "Distributed interferenceaware energy-efficient power optimization," IEEE Trans. Wireless Commun., vol. 10, no. 4, pp. 1323-1333, Apr. 2011.

[9] G. Bacci, E. V. Belmega, and L. Sanguinetti, "Distributed energyefficient power and subcarrier allocation for OFDMA-based small cells," in IEEE Intl. Conf. Commun. (ICC) Workshop on Small Cell and 5G Networks, Sydney, Australia, Jun. 2014, submitted.

[10] R. Corless, G. Gonnet, D. Hare, D. Jeffrey, and D. Knuth, "On the Lambert W function," Advances in Computational Mathematics, vol. 5, pp. 329-359, 1996.

[11] J. M. Cioffi, "A multicarrier primer," Amati Communications Corporation and Stanford University, Tech. Rep. 408-257-1717, 1991.

[12] G. Miao, N. Himayat, and G. Li, "Energy-efficient link adaptation in frequency-selective channels," IEEE Trans. Commun., vol. 58, no. 2, pp. 545-554, Feb. 2010.

[13] C. Y. Wong, R. S. Cheng, K. B. Letaief, and R. D. Murch, "Multiuser OFDM with adaptive subcarrier, bit, and power allocation," IEEE $J$. Select. Areas Commun., vol. 17, no. 10, pp. 1747-1758, Oct. 1999.

[14] H. Tabassum, Z. Dawy, and M. S. Alouini, "Sum rate maximization in the uplink of multi-cell OFDMA networks," in Intl. Wireless Commun. and Mobile Computing Conf. (IWCMC), Istanbul, Turkey, Jul. 2011.

[15] F. Facchinei and C. Kanzow, "Generalized Nash equilibrium problems," Quarterly J. Operations Research, vol. 5, no. 3, pp. 173-210, Sep. 2007.

[16] G. Bacci, L. Sanguinetti, M. Luise, and H. V. Poor, "Energy-efficient power control for contention-based synchronization in OFDMA systems with discrete powers and limited feedback," EURASIP J. Wireless Commun. and Networking (JWCN), vol. 2013, no. 1, Jul. 2013.

[17] W. Dinkelbach, "On nonlinear fractional programming," Management Science, vol. 13, no. 7, pp. 492-498, Mar. 1967.

[18] G. Scutari, D. Palomar, and S. Barbarossa, "Optimal linear precoding strategies for wideband non-cooperative systems based on game theory - Part II: Algorithms," IEEE Trans. Signal Process., vol. 56, no. 3, pp. 1250 - 1267, March 2008.

[19] S. Verdú, "On channel capacity per unit cost," IEEE Trans. Information Theory, vol. 36, no. 5, pp. 1019-1030, Sep. 1990. 